\title{
LA EVALUACIÓN DEL APRENDIZAJE Y SU INFLUENCIA EN EL COMPORTAMIENTO ESTRATÉGICO DEL ESTUDIANTE UNIVERSITARIO
}

\author{
Fermín Navaridas Nalda \\ Universidad de La Rioja
}

\begin{abstract}
RESUMEN. El desarrollo de líneas de investigación en/desde la propia práctica educativa es un elemento clave para poder establecer propuestas serias y rigurosas que permitan mejorar la calidad de la enseñanza superior en nuestros centros. En este marco, son numerosos los estudios que ponen de relieve el fuerte impacto de los factores personales y del propio contexto educativo sobre la calidad del aprendizaje universitario.

De acuerdo con todo ello, el presente trabajo centra su atención en el contexto singular de la enseñanza universitaria, abordándose el efecto de los procedimientos de evaluación del aprendizaje sobre las estrategias que adoptan los estudiantes durante su actividad de estudio. Las conclusiones a las que se llega vienen a corroborar la influencia de los sistemas de evaluación en los procesos de aprendizaje universitario y, en última instancia, en la calidad de los resultados obtenidos. Desde esta óptica, la evaluación de los alumnos puede ser tratada como un indicador de la calidad de la educación superior.
\end{abstract}

ABSTRACT. The development of lines of investigation from the same educational practice is a key to be able to establish serious and rigorous proposals that allow to improve the quality of the higher education in our centers. In this frame, there are numerous studies that emphasize the strong impact of the personal factors and of the same educational context on the quality of the university learning.

In agreement with all this, the present for paper centres its attention on the singular framework of the university education, with an approac on the effect of the assessment procedures of the learning on the strategies that the students adopt during their activity of study. The conclusions corroborate the influence of the assessment systems in the processes of university learning and, in last instance, in the quality of the obtained results. From this optics, the assessment of the pupils can be treated as an indicator of the quality of the education.

\section{Introducción}

Sin duda alguna, el cambio y la innovación de la enseñanza superior se han convertido en la actualidad en temas primordiales de debate universitario'. Entre los

1. Véanse, por ejemplo, las recomendaciones formuladas por los participantes en la Conferencia Mundial sobre la Educación Superior en el Siglo XXI (UNESCO, 1998) a fin de garantizar la excelencia 
aspectos que han suscitado este proceso activo de autorrevisión sobre la propia didáctica universitaria destacan, entre otros, la revolución tecnológica que acontece de una manera continua en los distintos sectores sociales y profesionales de nuestro entorno, el desarrollo exponencial y la diversificación del conocimiento, la creación de comunidades educativas cada vez más multiculturales y pluriétnicas ${ }^{2}$, así como la creciente preocupación gubernamental por acreditar la calidad del aprendizaje en «el espacio educativo europeo».

En este contexto de cambio y al amparo del nuevo paradigma educativo ${ }^{3}$ se proyecta la realización del presente trabajo, con el fin de contribuir a una mayor comprensión de la actividad didáctica e introducir propuestas de mejora en los procesos de enseñanza-aprendizaje que tienen lugar en el aula universitaria.

La razón que justifica la línea metodológica adoptada (Fig.1) se fundamenta en distintos estudios y experiencias llevadas a cabo en esta parcela de la realidad educativa investigada (Martón y Säljö, 1976; Entwistle, 1988; Biggs, 1989; Hernández Pina y cols. 1990; Printrich y cols., 1991; Alonso García, 1992; Mayor, Suengas y Marqués, 1993; Hernández Pina, 1996; Tejedor, 1998; Roces y cols. 1999; Justicia y Fuente, 1999; Buendía y Olmedo, 2000; Alonso Tapia, 2001). De acuerdo con los resultados obtenidos en estos trabajos, somos conscientes del numeroso conjunto de variables sobre las que incidir para alcanzar un mejor conocimiento y comprensión de la actividad didáctica en el aula universitaria: variables relacionadas con las características personales de los propios sujetos o agentes educativos investigados (edad, percepción, conocimientos previos, objetivos personales, motivaciones, modos preferentes de aprender, etc.); variables relacionadas con el contexto donde se desarrolla la actividad docente (infraestuctura, recursos didácticos, clima sociorrelacional de la clase, plan de estudios, contenido curricular, sistema de evaluación, etc.); variables relacionadas con el proceso (tarea, tipo de estrategia requerida, procedimientos utilizados, etc.); variables relacionadas con el producto (tipo de aprendizaje, rendimiento académico, nivel de satisfacción, impacto en el contexto socio-laboral, etc.). Entendemos que estos factores personales, socioambientales y propios de la actividad que inciden en el proceso de enseñanza-aprendizaje se encuentran interrelacionados unos con otros, convirtiéndose en un complejo sistema interactivo y singular de cada contexto.

Concretando más la situación de partida, nuestra investigación se centra en la evaluación del aprendizaje (variables presagio) y su influencia en las estrategias de apren-

de la enseñanza universitaria. En esta misma línea, resulta interesante analizar el impacto generado en los centros de enseñanza superior europeos la Declaración Conjunta de la Reunión de Ministros Europeos de Educación, celebrada en Bolonia durante el mes de junio del año 1999. Sobre este particular y su repercusión en la Armonización de las Estructuras Educativas de Europa, puede recabarse más información en la siguiente dirección electrónica: http://europa.eu.int/comm/education/tuning_es.html

2. En este sentido, un ejemplo de multiculturalidad universitaria lo tendríamos a partir de las acciones Erasmus llevadas a cabo dentro del marco del Programa Sócrates, en el que se ha implantado un Sistema Europeo de Transferencia de Créditos (ECTS) para favorecer la movilidad de estudiantes y el intercambio de experiencias en un nuevo entorno de aprendizaje compartido.

3. Frente a la concepción tradicional del profesor universitario que trata de desarrollar, codificar y transmitir a los estudiantes cuantos más conocimientos mejor (Benedito, Ferrer y Ferreres, 1995: 133), aparece un nuevo paradigma que pone de relieve la necesidad de invertir el centro de atención de la enseñanza al aprendizaje (Coll, Palacios y Marchesi, 1992; Zabalza, 2000). 


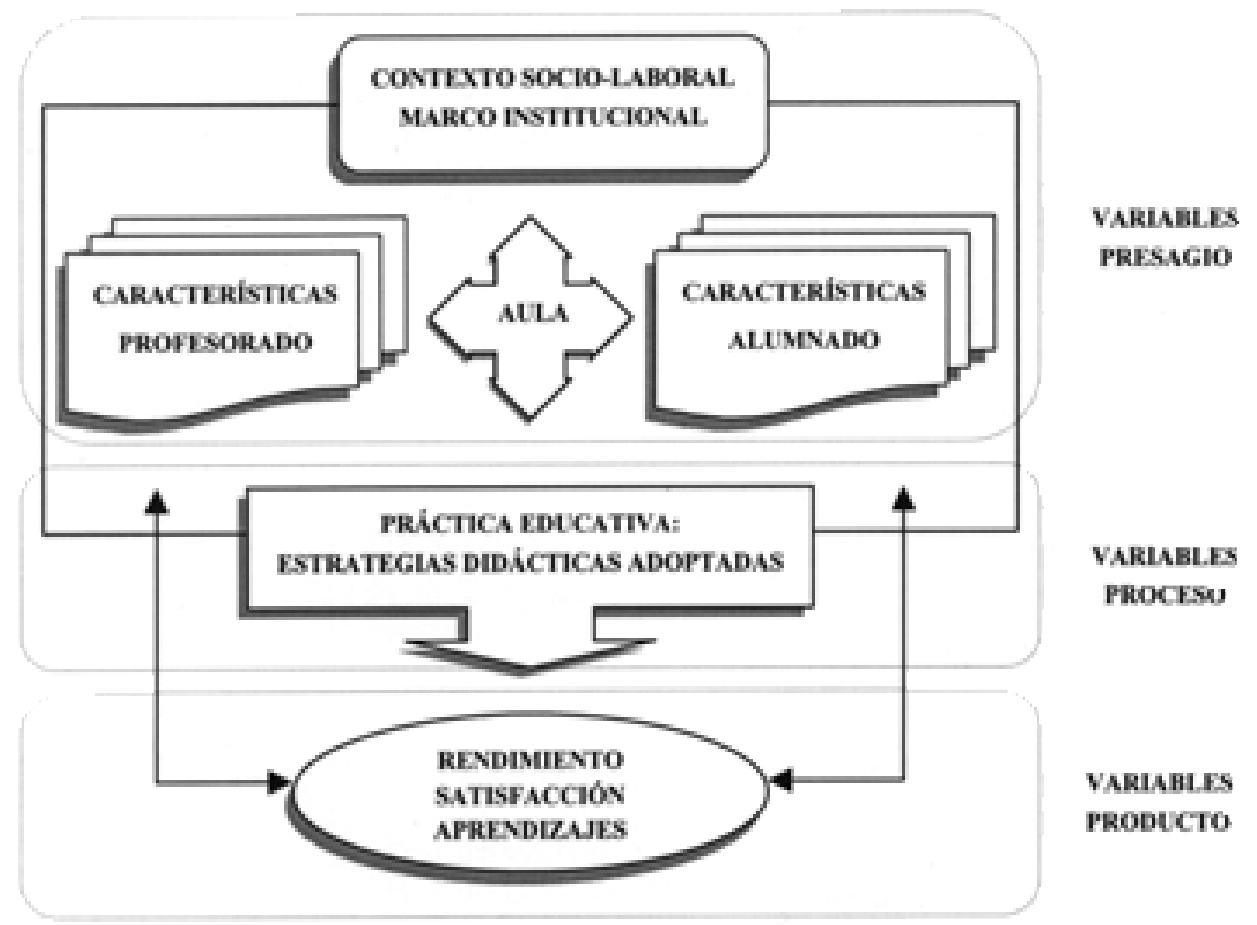

Figura 1. Modelo para el estudio de la actividad didáctica universitaria.

dizaje que adoptan los estudiantes durante su actividad de estudio en la Universidad (variables proceso). Desde estas coordenadas, si asumimos que el modo o los modos concretos de enfocar la evaluación condicionan el comportamiento estratégico del estudiante durante el proceso formativo (Hernández Pina, 2001), los resultados obtenidos deben ser considerados como elementos importantes para la reflexión docente sobre el tipo de aprendizaje promovido y la calidad de este aprendizaje en el aula universitaria (variables producto).

Abundando en esta línea, para Morales (1998: 24) "el cómo estudia el alumno no es una cuestión trivial, porque el cómo estudia es lo que forma al alumno (o lo deforma). Un alumno estudiará de memoria o estudiará inteligentemente, procurando entender y relacionar, según sea el tipo de examen o pregunta esperado. El profesor no sólo controla qué hace el alumno en clase (escuchar, hacer un ejercicio, etc.) sino que además controla cómo estudia y trabaja en su casa o en otros lugares cuando prepara exámenes y tareas". En consecuencia la evaluación se constituye en sí misma en una estrategia docente sumamente eficaz para conseguir aprendizajes universitarios de calidad y, desde esta óptica, parece conveniente potenciar su investigación al objeto de recabar información sobre la que fundamentar decisiones futuras para la innovación y la mejora de la práctica educativa. 


\section{Delimitación del problema objeto de estudio: Cuestiones fundamentales}

Como ya hemos señalado con anterioridad, la «calidad de la enseñanza» se ha convertido en un tema de máxima preocupación e interés para todas las universidades europeas. Esta preocupación por mejorar la enseñanza en general y proporcionar una formación integral de calidad a los estudiantes, en particular, es también compartida por toda la comunidad universitaria riojana. Ahora bien, ¿cómo podemos mejorar nuestra práctica educativa? ¿Mejorar para qué y para quién?

Las nuevas tendencias de la teoría evaluadora en el marco de la educación superior están poniendo cada vez más de relieve la necesidad de establecer un proceso de mejora que vaya más allá de la simple evaluación de la función docente por parte de los estudiantes. Ahora bien, no tratamos de decir en ningún caso que la evaluación docente sea un procedimiento poco importante; así como tampoco tratamos de cuestionar su validez. Más bien, pensamos que por sí sola puede resultar insuficiente para estos fines. Por tanto, sin descartar otras medidas, consideramos importante enriquecerlas con nuevas formas e instrumentos de autorrevisión en/desde la propia práctica en el aula.

Así, por ejemplo, ¿por qué no pedir a esos mismos estudiantes que valoren su actividad discente? ¿Hasta qué punto puede resultar interesante al profesorado universitario conocer las estrategias de aprendizaje que se activan durante sus clases? ¿Cómo influyen los tipos o enfoques de la evaluación en el aprendizaje de los alumnos? ¿Qué hacen los alumnos cuando estudian? ¿Estudian para aprender o lo hacen para aprobar? ¿Por qué? Estas cuestiones suscitaron desde un primer momento nuestro interés por la investigación realizada.

En este contexto, $y$ atendiendo a las consideraciones planteadas, tratamos de poner en relación todos los datos recogidos para comprender mejor lo que sucede en nuestra institución educativa, buscar su significado $y$, de este modo, diseñar propuestas desde la propia acción con el propósito de mejorarla.

\section{Objetivos de la investigación}

Una forma de contribuir al cambio y a la innovación de la enseñanza superior en nuestros centros es «analizar la actividad didáctica desde la propia perspectiva de los sujetos que la protagonizan». Con la finalidad referida, nos proponemos en este trabajo de investigación los siguientes objetivos:

a) Identificar los modos preferentes de evaluar el aprendizaje en el aula universitaria.

b) Identificar las estrategias generales de aprendizaje que utilizan los estudiantes durante su actividad de estudio.

c) Determinar el grado de influencia de los modos preferentes de evaluar en las estrategias de aprendizaje que se activan durante el estudio universitario.

d) Sensibilizar a los agentes educativos implicados en la investigación hacia actuaciones de autorrevisión sobre la forma de proceder en los procesos de enseñanzaaprendizaje universitario. 


\section{Metodología de la investigación}

En este apartado describimos de forma concisa los sujetos investigados, el instrumento utilizado para recabar información, así como los distintos procedimientos aplicados para el análisis y tratamiento de los datos obtenidos durante el trabajo de campo.

\subsection{Sujetos participantes en la investigación}

La población de nuestro estudio está definida por los alumnos matriculados en materias de carácter obligatorio de primero y último curso de las titulaciones oficiales ofertadas por la Universidad de La Rioja. El número de estudiantes que respondieron al cuestionario administrado fue de 908 (el 45,15\% de la población investigada). De ellos, el 65,5\% (595) se encuentran matriculados en el Centro de Ciencias Humanas, Jurídicas y Sociales, mientras que el 34,5\% (313) restante cursa sus estudios en el Centro de Enseñanzas Científicas y Técnicas.

Con relación al curso donde se encuentran matriculados (Fig. 2), el 60\% (545) de los estudiantes encuestados son de primero y el $40 \%$ (363) de último curso. No obstante, y si tenemos presente las dos modalidades de titulaciones investigadas (por un lado, las Diplomaturas e Ingenierías Técnicas y, por otro, las Licenciaturas e Ingeniería Superior), hay que considerar que del conjunto total de estudiantes de último curso el $57 \%$ (206) son de cuarto y el $43 \%$ (157) corresponden a tercero.

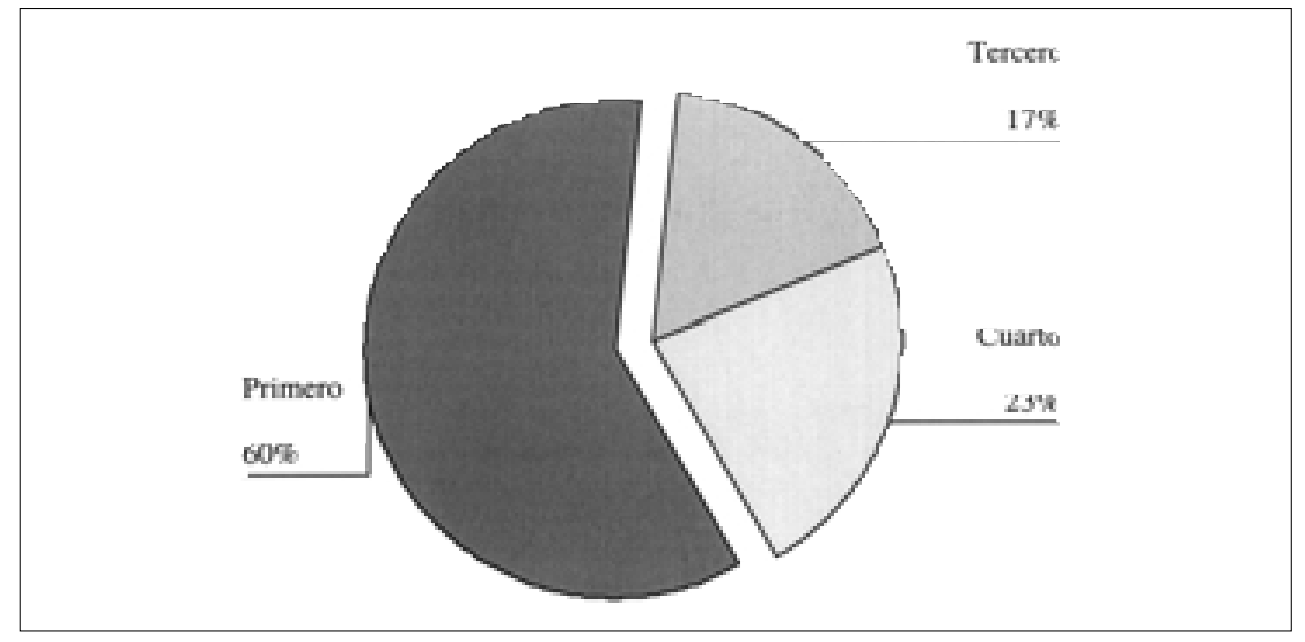

Figura 2. Distribución de la muestra por cursos

Como podemos observar en la Tabla 1, la muestra está formada por un 58,3\% de mujeres frente al $41,7 \%$ de hombres, siendo la media de edad para ambos grupos de 21,39 años. El $70 \%$ de los estudiantes participantes tienen edades comprendidas entre los 18 y 22 años, estando la moda en 19 años.

Haciendo un análisis por cursos, se puede apreciar que la media de edad es ligeramente superior a la que correspondería si se hubiesen ajustado totalmente a los 
períodos previstos en el sistema educativo universitario. Sin embargo, estos datos no se corresponden con los valores obtenidos a través de la mediana y la moda en cada uno de los casos estudiados. Así, por ejemplo, en el grupo de alumnos de primer curso (545), esta diferencia podría deberse a un pequeño grupo de estudiantes (35) mayores de 25 años que se incorporan más tarde de lo habitual al sistema.

Con todo, podríamos presumir un primer perfil general del estudiante investigado en la Universidad de La Rioja. A grandes rasgos, se trata de un alumno/a de unos 21 años de edad aproximadamente, dedicado/a exclusivamente al estudio y que, con bastante probabilidad, se encuentra cursando la carrera que eligió como primera opción tras finalizar las Enseñanza Medias. Su vía de acceso a la Universidad fue a través de la prueba de Selectividad, siendo su nota media en torno al 6,3.

\begin{tabular}{|c|c|c|c|}
\hline CARAC & CTERÍSTICAS & № & $\% *$ \\
\hline \multicolumn{4}{|l|}{ Sexo } \\
\hline & Hombre & 379 & 41,7 \\
\hline & Mujer & 529 & 58,3 \\
\hline \multicolumn{4}{|l|}{ Edad } \\
\hline & Menos de 23 años & 632 & 69,6 \\
\hline & De 23 a 27 & 253 & 27,8 \\
\hline & Más de 28 & 23 & 2,6 \\
\hline \multicolumn{4}{|c|}{ Vía de acceso a la Universidad } \\
\hline & Selectividad & 756 & 83,3 \\
\hline & C.O.U. & 31 & 3,4 \\
\hline & Formación Profesional & 78 & 8,6 \\
\hline & Mayores de 25 años & 3 & 0,3 \\
\hline & Otras titulaciones & 35 & 3,9 \\
\hline & NS/NC & 5 & 0,6 \\
\hline \multicolumn{4}{|l|}{ ¿Trabaja? } \\
\hline & No & 723 & 79,6 \\
\hline & Si & 185 & 20,4 \\
\hline
\end{tabular}

Tabla 1. Perfil sociodemográfico de los entrevistados. 


\subsection{Instrumento utilizado en la investigación}

El instrumento utilizado para llevar a cabo el proceso de recogida de datos es el CEAUR-II: «Cuestionario sobre estrategias de aprendizaje en el aula universitaria ${ }^{4}$ (Navaridas, 2001). Este Cuestionario consta de dos partes bien diferenciadas. Una primera donde se formulan cuestiones relativas a las variables presagio (características personales del alumnado y propias del contexto de enseñanza) que influyen sobre el comportamiento estratégico del estudiante universitario (véase un ejemplo en la Tabla 2 que se adjunta).

A continuación encontrarás distintos instrumentos de medida que pueden estar utilizando los profesores para la evaluación de tus aprendizajes.

En general, ¿cuál es la frecuencia con que se utilizan durante este curso concreto?

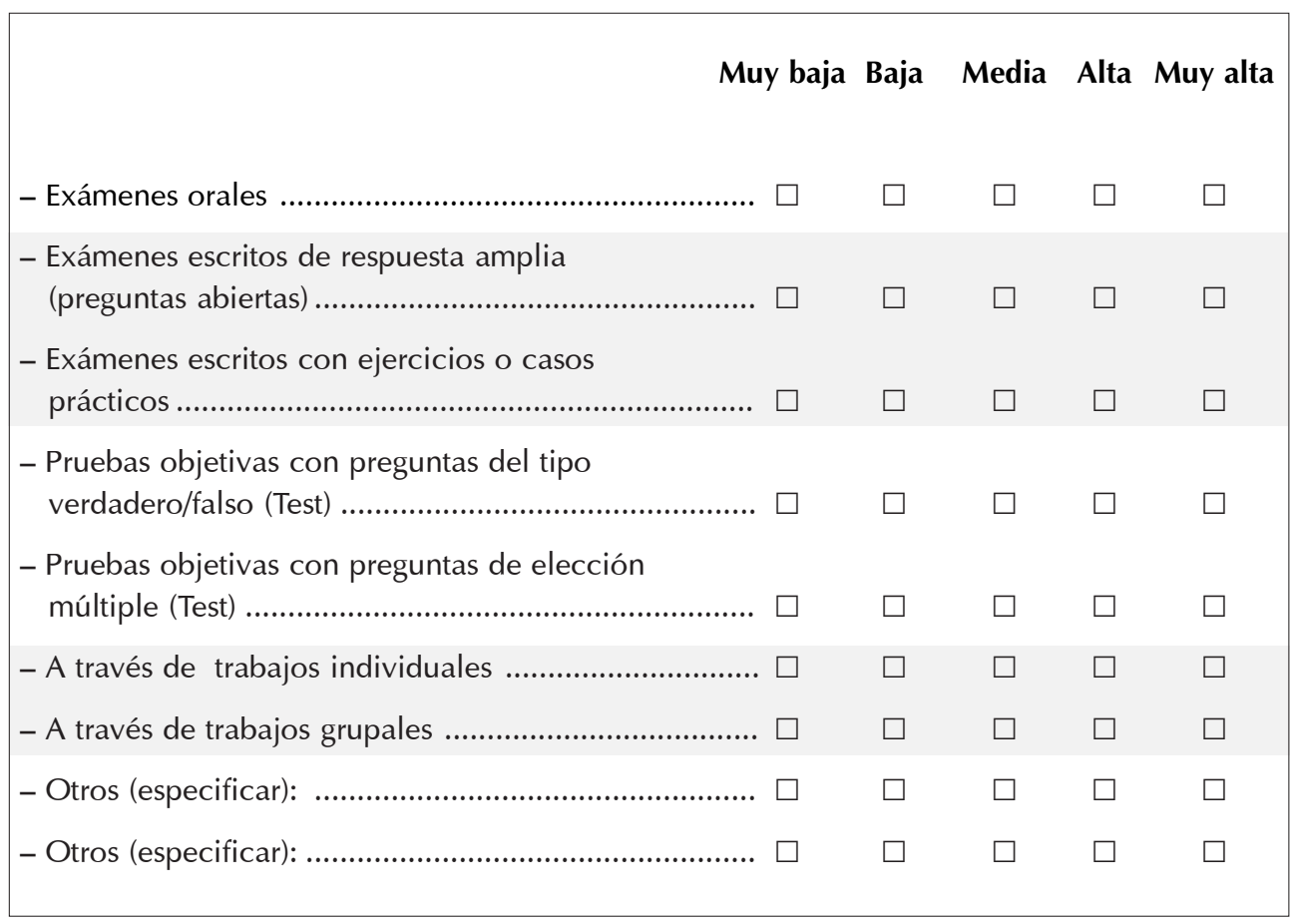

Tabla 2. Escala de intervalo contemplada en la primera parte del Cuestionario.

La segunda parte consta de 45 ítems o afirmaciones declaradas en términos de estrategias que utilizan los estudiantes con mayor o menor frecuencia durante su pro-

4. Cuestionario que por limitaciones de espacio evitamos reproducir en este trabajo, pero cuyo proceso de elaboración, formato, estructura y características fundamentales que lo determinan puede consultarse en la referencia bibliográfica citada. 
ceso de aprendizaje. Los primeros 20 ítems hacen referencia a estrategias generales de naturaleza cognitiva en el sujeto que aprende (adquisición, codificación y recuperación del material informativo). Los ítems 21 a 27, ambos inclusive, tratan de enjuiciar aspectos relativos a las estrategias metacognitivas (utilizadas durante el proceso de estudio para planificar, regular y controlar el propio funcionamiento cognitivo). Por último incluye una serie de ítems (del 28 al 45) relativos a la variable denominada estrategias de apoyo, orientadas a mantener un estado cognitivo adecuado durante la actividad formativa (motivacionales, afectivas y actitudinales).

\subsection{Análisis y tratamiento de los datos: Resultados}

La información recogida mediante el cuestionario ha sido analizada de forma cuantitativa en base a estadísticos descriptivos y multivariantes. Así, los procedimientos utilizados en el tratamiento de los datos fueron seleccionados de acuerdo con dos criterios básicos:

- Carácter no métrico (medidas en escala nominal u ordinal) o métrico (medidas en escalas de intervalo o de razón) de las variables.

- Número de variables que intervienen en los distintos tratamientos estadísticos, dando lugar a procedimientos univariados, bivariados y multivariados.

En general, y a la vista de los resultados obtenidos (Fig. 3), podemos destacar que los exámenes escritos con ejercicios o casos prácticos es el instrumento de medida más utilizado por los profesores para la evaluación de los aprendizajes $(\bar{X}=3,72)$. Con menor frecuencia aparece la evaluación a través de exámenes escritos de respuesta amplia $(\bar{X}=3,33)$, los trabajos individuales $(\bar{X}=2,63)$ y las pruebas objetivas con preguntas de elección múltiple ( $\bar{X}=2,50)$. Asimismo, como puede comprobarse en la Figura 3, los exámenes orales son los menos utilizados por el profesorado docente para la evaluación de los aprendizajes en el aula universitaria investigada $(\bar{X}=1,23)$.

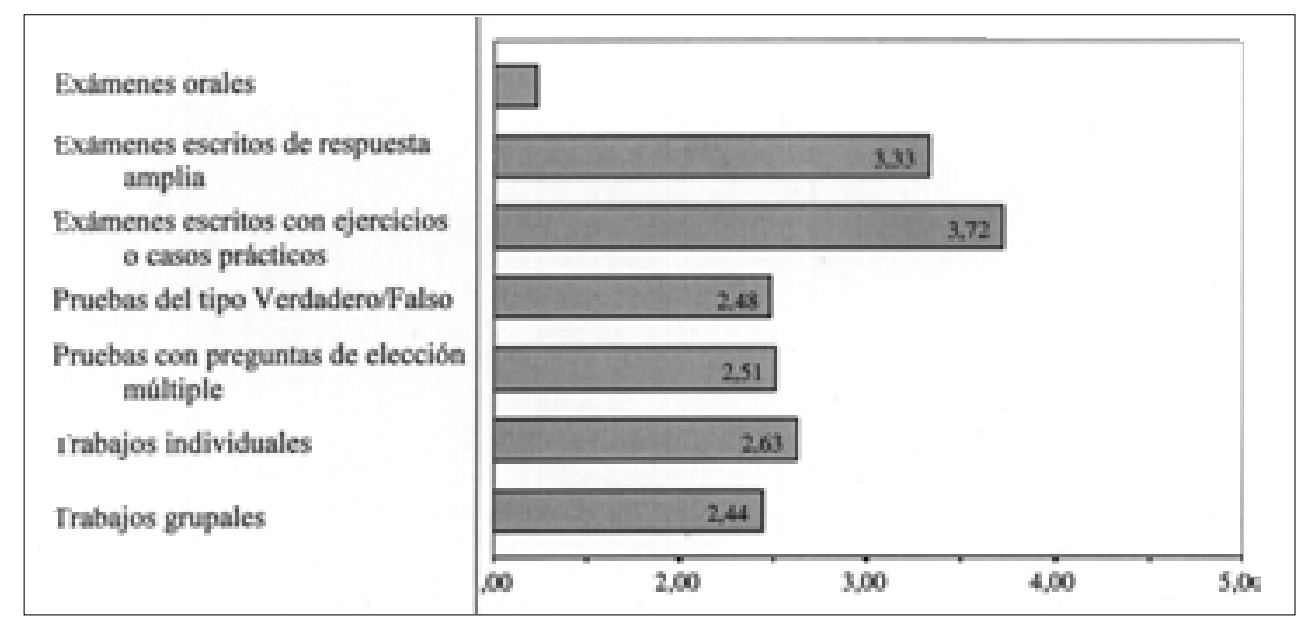

Fig. 3. Medias de frecuencias de utilización de las diferentes formas de evaluación en la Universidad de La Rioja. 
Por titulaciones (Fig. 4), la evaluación a través de exámenes escritos con ejercicios o casos prácticos destaca más en carreras consideradas de "ciencias" como Matemáticas, Química o Ingeniería Industrial. En Magisterio, Filología y Humanidades aparecen con mayor frecuencia los exámenes escritos de respuesta amplia (preguntas abiertas).

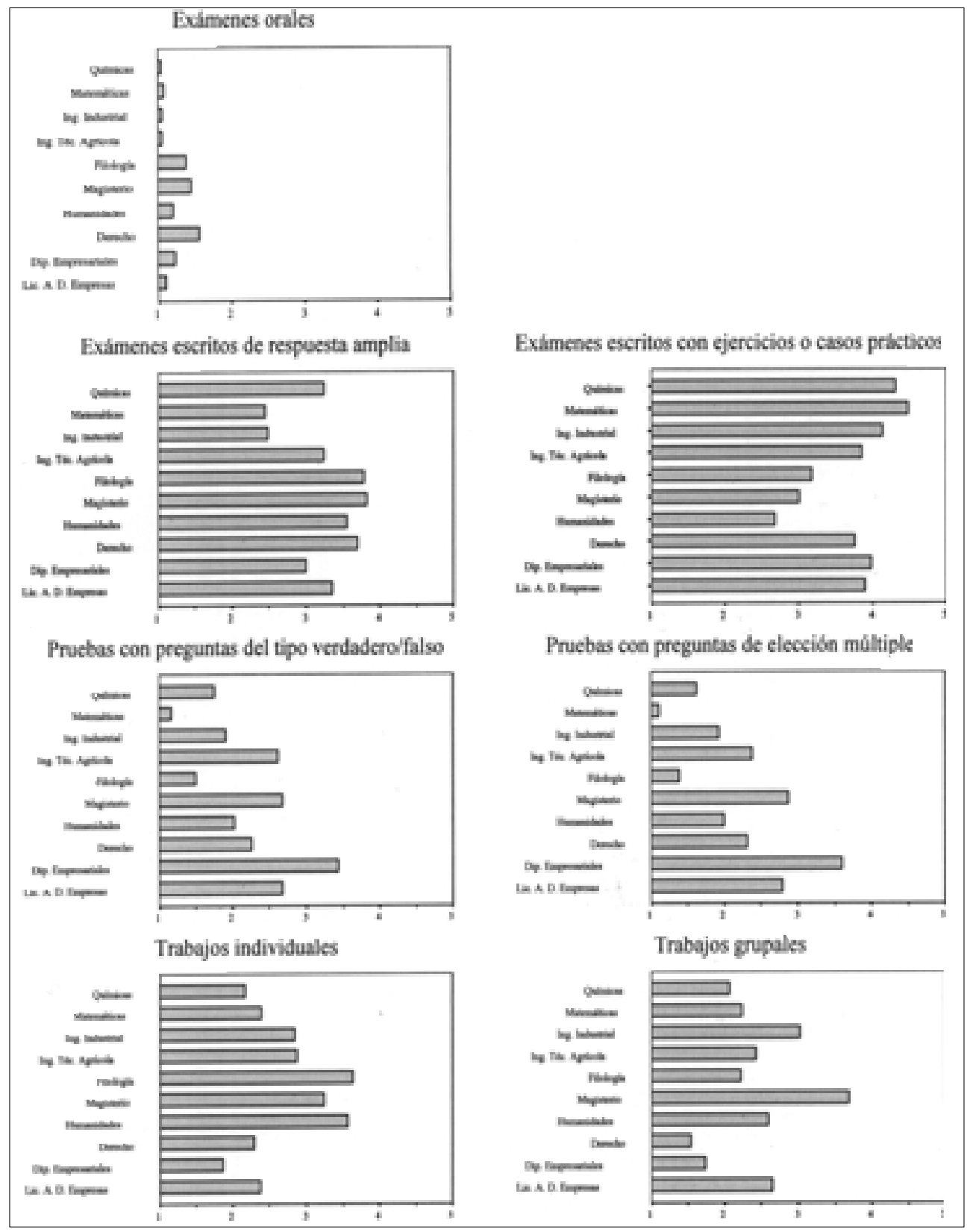

Fig. 4. Frecuencias de utilización de las diferentes formas de evaluación por titulaciones. 
Por otra parte, observamos (Fig. 5) que las estrategias cognitivas son las más utilizadas $(\bar{X}=3,32)$ durante los procesos de aprendizaje de los sujetos investigados, mientras que el nivel medio de utilización de los otros tipos de estrategias es relativamente menor: estrategias de apoyo al procesamiento de información $(\overline{\mathrm{X}}=3,29)$ y estrategias metacognitivas $(\bar{X}=3,20)$; a un nivel de significación de $p$-valor $=0,009$ y $p$-valor $=0,000$ respectivamente.

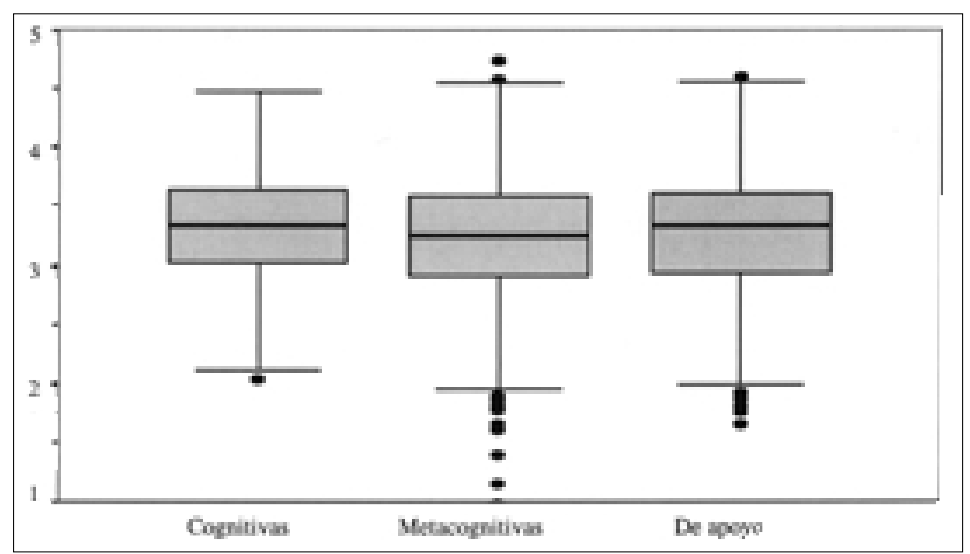

Fig. 5. Utilización de las estrategias de aprendizaje durante la actividad de estudio en la Universidad de La Rioja.

Dentro de las estrategias de naturaleza cognitiva (Fig. 6), los estudiantes activan con mayor frecuencia estrategias para la adquisición $(\bar{X}=3,47)$ de unidades de información (copiar, explorar, subrayar...) y recuperación $(\bar{X}=3,45)$ del material objeto de aprendizaje (búsqueda de indicios, libre asociación...) que estrategias de codificación $(\bar{X}=3,06)$ o almacenamiento significativo de la información en su estructura cognitiva (procedimientos mnemotécnicos, mapas conceptuales, clasificaciones, metáforas, analogías, esquemas, resúmenes...), a un nivel de significación p-valor=0,000.

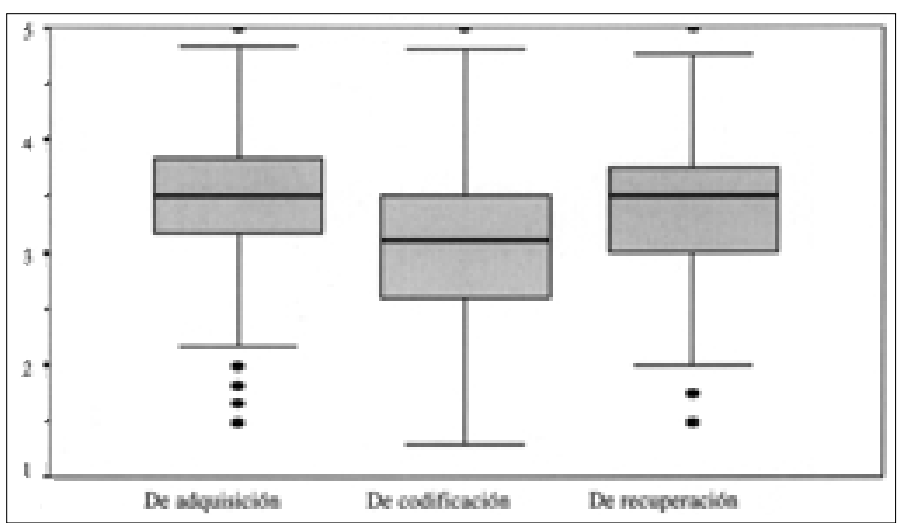

Fig. 6. Niveles de utilización de las Estrategias de Aprendizaje Cognitivas: Adquisición, Codificación y Recuperación. 
En cuanto a las estrategias metacognitivas (Fig. 7), encontramos que existen diferencias estadísticamente significativas ( $p$-valor $=0,000$ ) de acuerdo con los procesos a los que sirven: los estudiantes universitarios investigados utilizan más estrategias de autoconocimiento $(\bar{X}=3,42)$ que estrategias para la planificación o control de su propia actividad cognitiva $(\bar{X}=3,00)$.

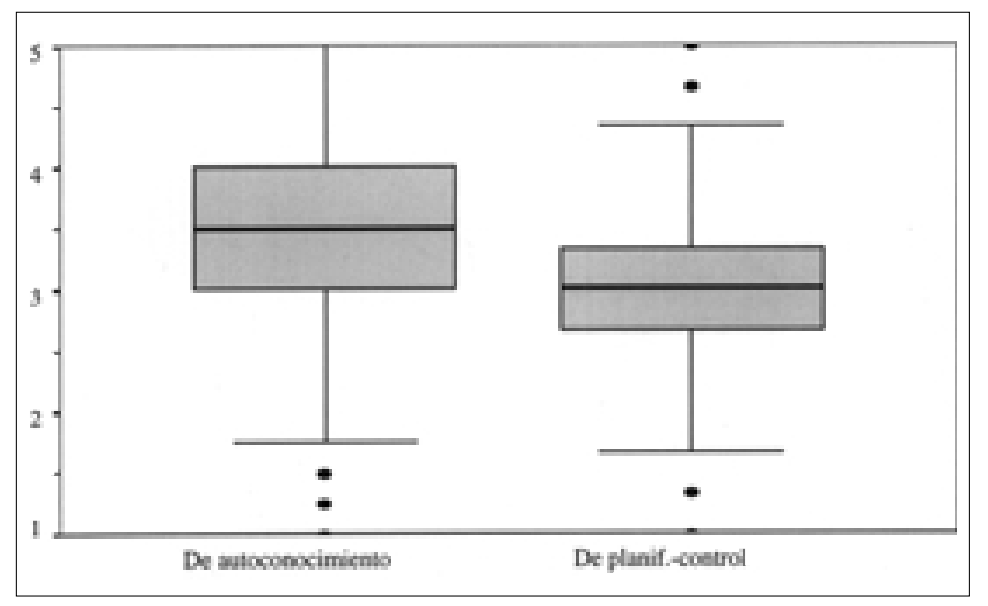

Fig. 7. Niveles de utilización de las Estrategias Metacognitivas: Autoconocimiento y Planificación-Control.

Con respecto a las estrategias de apoyo adoptadas por los estudiantes para mantener un estado cognitivo adecuado durante su actividad de estudio, hemos podido constatar (Fig. 8) que las motivacionales $(\bar{X}=3,52)$ se utilizan significativamente más ( $p$-valor $=0,000)$ que las actitudinales $(\bar{X}=3,26)$ y las afectivas $(\bar{X}=3,08)$.

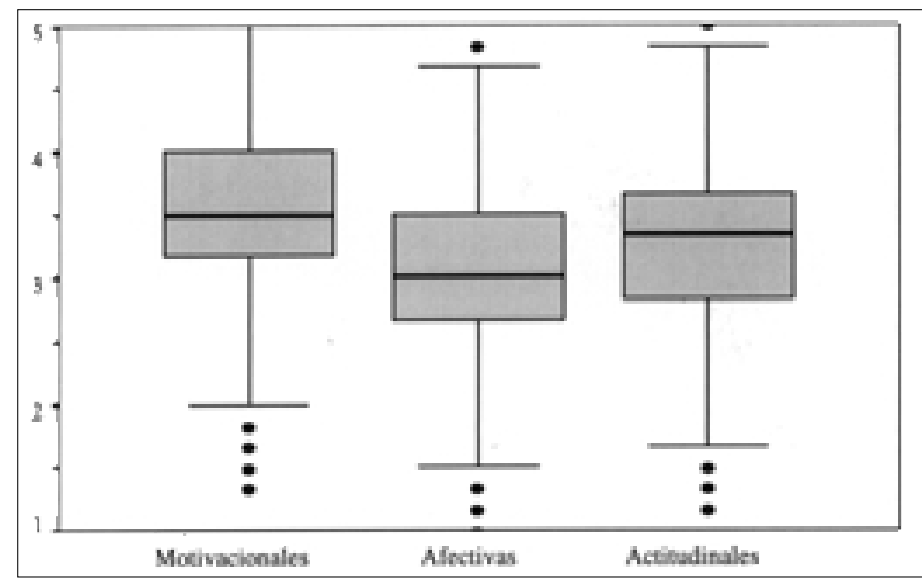

Fig. 8. Niveles de utilización de las Estrategias de Apoyo: Motivacionales, Afectivas y Actitudinales. 
Finalmente, una vez identificadas las estrategias generales de aprendizaje durante la actividad de estudio, tratamos de comprobar hasta qué punto influyen en su utilización los distintos modos de evaluar adoptados por el profesorado en el aula universitaria. En general, y a la vista de los resultados obtenidos en el tratamiento estadístico (Figs. 9,10 y 11), podemos afirmar que los estudiantes universitarios utilizan más estrategias de aprendizaje cuando se les evalúa asiduamente (frecuencia Alta / Muy alta) mediante trabajos de carácter individual o grupal.

\begin{tabular}{|c|c|c|c|c|}
\hline MODOS DE EVALUAR & ESTRATEGIAS COGNITIVAS & $\begin{array}{c}\mathbf{X}_{2} \\
\text { (Fr. Baja) } \\
\end{array}$ & $\begin{array}{c}\mathrm{X}_{2} \\
\text { (Fr. alta) } \\
\\
\end{array}$ & $\begin{array}{c}\text { Sig. } \\
\text { p-valer }\end{array}$ \\
\hline Eximenes orales & & 3,33 & 3,13 & 0,184 \\
\hline Eximenes escritos abiertos & & 3,27 & 3,39 & 0,000 \\
\hline Eximenes escritos con ejercicios & & 3,33 & 3,33 & 0,848 \\
\hline Pruebas ob, verdadero / falso & & 3,32 & 3,37 & 0,183 \\
\hline Prucbas ob. elección múltiple & & 3,31 & 3,39 & 0,024 \\
\hline Trabajos individuales & & 3,29 & 3,46 & 0,000 \\
\hline \multirow[t]{2}{*}{ Trabajos grupales } & & \multirow[t]{2}{*}{3,30} & \multirow[t]{2}{*}{3,43} & \multirow[t]{2}{*}{0,001} \\
\hline & 2 & & & \\
\hline
\end{tabular}

Fig. 9. Utilización de las estrategias cognitivas en función de los distintos modos de evaluar los aprendizajes en la Universidad de La Rioja.

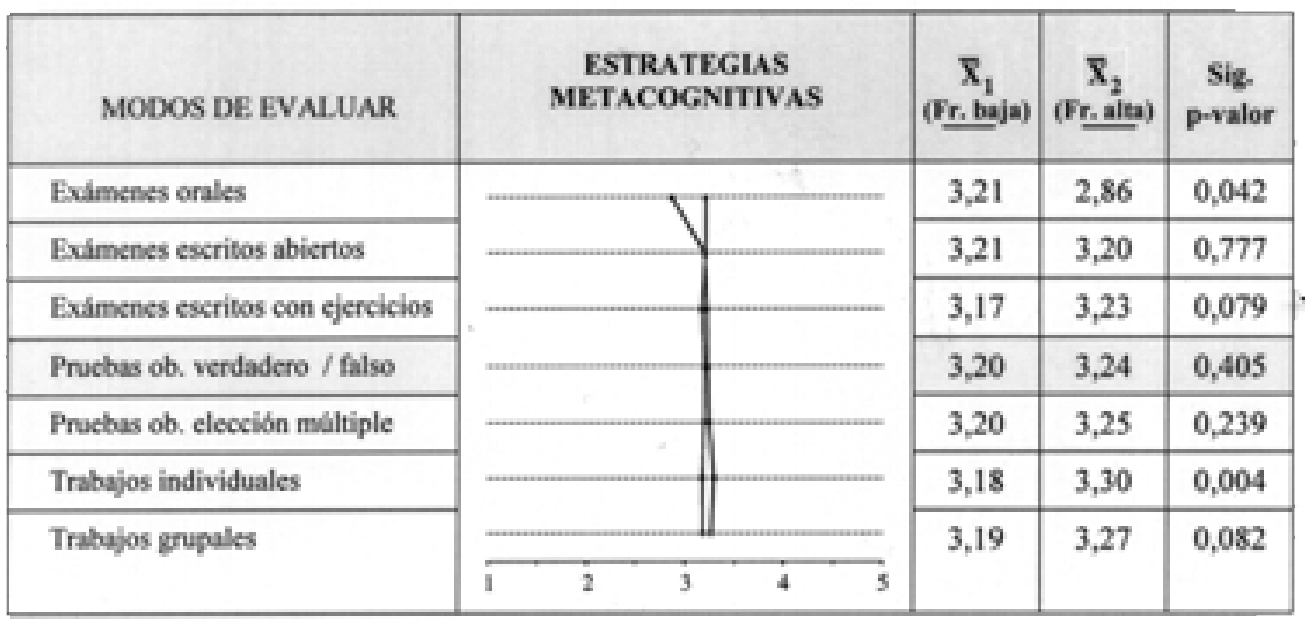

Fig. 10. Utilización de las estrategias metacognitivas en función de los distintos modos de evaluar los aprendizajes en la Universidad de La Rioja. 


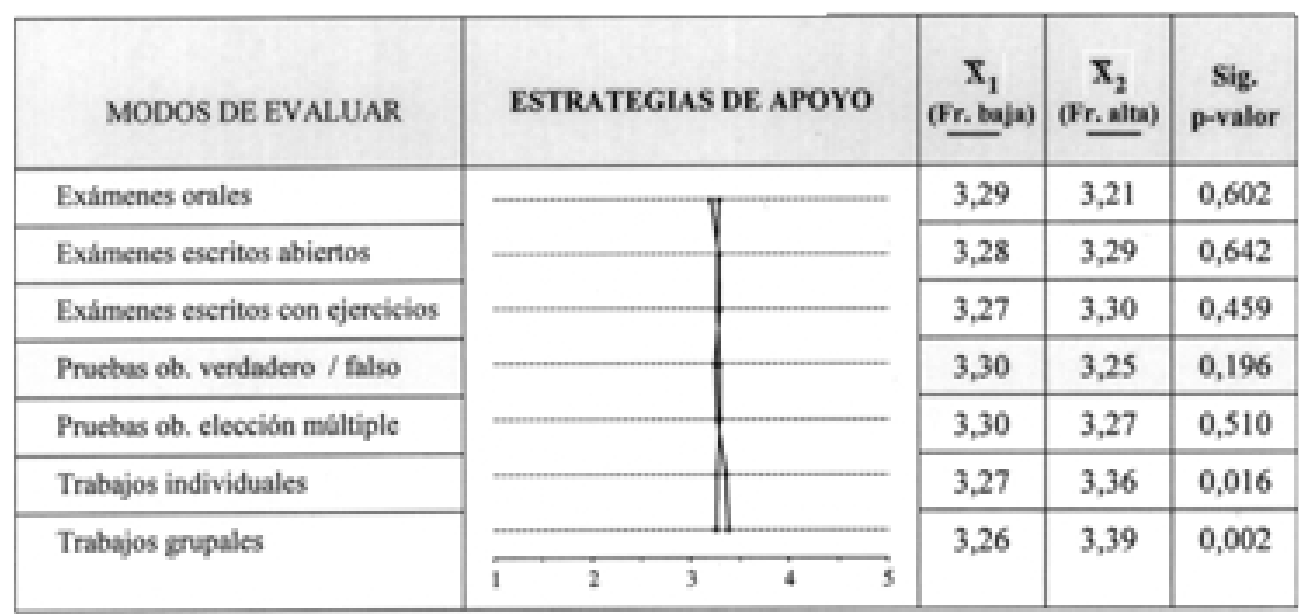

Fig. 11. Utilización de las estrategias de apoyo al procesamiento en función de los distintos modos de evaluar los aprendizajes en la Universidad de La Rioja.

Del mismo modo, podemos observar (Fig. 9) diferencias estadísticamente muy significativas ( $p$-valor $=0,000$ ) en la puntuación total obtenida en las estrategias de naturaleza cognitiva según los alumnos realicen con mayor o menor frecuencia exámenes escritos con preguntas abiertas (donde tiene que presentar una información organizada, ordenar, explicar, mostrar comprensión...). En este caso, la media obtenida en estrategias cognitivas por los alumnos evaluados de forma frecuente (Alta /Muy alta) a través de preguntas abiertas es mayor $\left(\overline{\mathrm{X}}_{2}=3,39\right)$ que en aquellos casos donde no deben afrontar con tanta frecuencia (Muy baja / Baja / Media) este tipo de pruebas

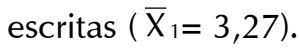

Es de destacar, también, el hecho de que los alumnos que son evaluados con relativa frecuencia (Alta / Muy alta) a través de exámenes escritos que incluyen ejercicios o casos prácticos utilizan más estrategias de naturaleza metacognitiva $\left(\bar{X}_{2}=3,23\right)$ que en aquellos casos donde esta modalidad para la evaluación del aprendizaje es menos frecuente (Muy baja / Baja / Media) ( $\left.\bar{X}_{1}=3,17\right)$, con un nivel de significación observado ó p-valor=0,079 (Fig. 10).

\section{Conclusiones y valoración global del estudio}

Una vez presentados los resultados de la investigación, es el momento de realizar un esfuerzo final de síntesis al objeto de extraer las conclusiones más significativas de este trabajo desarrollado en la Universidad de La Rioja.

En primer lugar, podríamos afirmar que el entorno de la evaluación condiciona la forma de aprender del alumnado. Es decir, las estrategias generales que utilizan los estudiantes en el aprendizaje que tiene lugar durante su actividad de estudio dependen en buena medida de los exámenes esperados o imaginados en sus profesores. 
Así, por ejemplo, los estudiantes adoptan una actitud más estratégica frente al aprendizaje cuando se les evalúa asiduamente a través de trabajos individuales o grupales: utilizan más estrategias cognitivas (para buscar, seleccionar, elaborar, organizar y recuperar el material informativo), estrategias metacognitivas (para planificar, dirigir y modificar el funcionamiento cognitivo) y de apoyo al procesamiento (para regular su esfuerzo, de búsqueda de ayuda, mediante el aprendizaje con otros).

Desde esta óptica, los trabajos en equipo como sistema de evaluación formativa en el aula univesitaria, ofrecen una gran oportunidad didáctica para que los estudiantes puedan construir aprendizajes significativos para su vida personal y profesional futura (no olvidemos que su utilidad parece ir más allá del mero ámbito cognitivo: hábitos de trabajo, autorregulación del esfuerzo, cooperación, responsabilidad, organización del tiempo, búsqueda de ayuda...). De este modo, y de acuerdo con las necesidades que se plantean en el nuevo marco de la educación superior, deberíamos reconsiderar además del componente académico de nuestra enseñanza una "dimensión humana" que también prepara para la posterior actuación profesional y desarrollo de los estudiantes.

Por otra parte, cuando la evaluación se centra en exámenes escritos con preguntas abiertas (explicar con cierta amplitud determinados aspectos del material informativo, organizar ideas, presentar una estructura, exponer las ideas principales, mostrar comprensión...) los alumnos utilizan más estrategias de naturaleza cognitiva durante el proceso de estudio.

Dentro del grupo de estrategia cognitivas, los estudiantes activan con mayor frecuencia procedimientos para la adquisición de información (copiar las exposiciones del profesor, subrayar las ideas importantes, recitar la información relevante) y la recuperación del material informativo almacenado en la memoria (búsqueda de indicios en los exámenes, claves, planificación de respuestas, libre asociación), frente a otros procedimientos específicos de codificación orientados a elaborar y organizar la información de forma significativa en su estructura cognitiva (mnemotécnicos, autoexplicaciones personales, metáforas, analogías, clasificaciones, mapas conceptuales, esquemas, resúmenes). No es casual por tanto que las estrategias metacognitivas (entendidas como secuencias de procedimientos dirigidos a reflexionar sobre los propios procesos de comprensión), sean también las menos utilizadas por los estudiantes investigados.

Estos datos vienen a confirmar que la actividad del estudiante universitario se ajusta en gran medida al tipo de evaluación predominante durante la actividad docente. Corrobora esto que decimos, el hecho de que los alumnos que son evaluados con relativa frecuencia a través de exámenes escritos con ejercicios o casos prácticos utilizan más estrategias de naturaleza metacognitiva que en aquellas situaciones donde esta modalidad de examen no es tan frecuente.

Finalmente, y al objeto de optimizar la actividad didáctica en el aula universitaria, sería necesario analizar el grado de influencia de otras variables de naturaleza personal (motivaciones, percepciones, conocimientos previos, etc.) y específicas del contexto docente (estrategias de enseñanza, clima sociorrelacional de la clase, tipo de asignaturas, etc.) obviadas en este estudio de caso. No obstante, estamos convencidos que los resultados aportados en este trabajo suponen en sí mismos un proceso de 
reflexión importante para la puesta en marcha de acciones concretas de mejora en nuestros centros de enseñanza.

\section{Referencias bibliográficas}

ALONSO GARCÍA, C. M. (1992): Análisis y Diagnóstico de los Estilos de Aprendizaje en Estudiantes Universitarios. Tesis doctoral. Facultad de Filosofía y Ciencias de la Educación. Universidad Complutense de Madrid.

ALONSO TAPIA, J. (2001): "Motivación y estrategias de aprendizaje. Principios para su mejora en alumnos universitarios". En GARCÍA-VALCÁRCEL, A. (COORD.): Didáctica universitaria. Madrid: La Muralla.

BENEDITO, V; FERRER, V. y FERRERES, V. (1995): La formación universitaria a debate. Barcelona: Publicacions de la Universitat de Barcelona.

BIGGS, J.B. (1989): "Approaches to the Enhancement of Tertiary Teaching". En Higher Education Research and Development, 8 (1), págs. 7-25.

BUENDÍA, L. y OLMEDO, M. (2000): "Estrategias de aprendizaje y procesos de evaluación en la educación universitaria". En Bordón, 52 (2), págs. 151-163.

COLL, C; PALACIOS, J. y MARCHESI, A. (Eds.) (1992): Desarrollo Psicológico y Educación II. Psicología de la Educación. Madrid: Alianza.

ENTWISTLE, N. (1988): La comprensión del aprendizaje en el aula. Barcelona: Paidós-MEC.

HERNÁNDEZ PINA, F. (1996): "La evaluación de los alumnos en el contexto de la evaluación de la calidad de las universidades". En Revista de Investigación Educativa, 14 (2), págs. 25-50

HERNÁNDEZ PINA, F. (2001): "La calidad de la enseñanza y el aprendizaje en educación superior". En Revista de Investigación Educativa, 19, 2, págs.463-489.

HERNÁNDEZ PINA, F. y cols. (1990): "Enfoques de aprendizaje universitario como base para el diagnóstico de necesidades". En Revista de Investigación Educativa, 8 (16), págs 239-253.

JUSTICIA, F. y FUENTE, J. de la (1999): "Análisis factorial de las Escalas ACRA en una muestra de alumnos universitarios". En Mente y Conducta en Situación Educativa. Revista Electrónica del Departamento de Psicología de la Universidad de Valladolid, Vol. I, 1, págs. 51-50.

MARTON, F. y SÄLJÖ, R. (1976): "On qualitative differences in learning: I. Outcome and process". En British Journal of Educational Psychology, 46, págs. 4-11.

MAYOR, J.; SUENGAS, A. y GONZÁLEZ, J. (1993): Estrategias metacognitivas. Aprender a aprender $y$ aprender a pensar. Madrid: Síntesis.

MORALES, P. (1998): Evaluación y aprendizajes de calidad. Guatemala: Universidad Rafael Landívar.

NAVARIDAS, F. (2001): Análisis de las estrategias de enseñanza y aprendizaje en La Universidad de La Rioja: Propuesta de mejora. Tesis doctoral. Facultad de Educa- 
ción. Departamento de Didáctica, Organización Escolar y Didácticas Específicas. Universidad Nacional de Educación a Distancia.

PINTRICH, P.R.; SMITH, D.A.; GARCÍA, T. y MCKEACHIE, W.J. (1991): A Manual for the Use of the Motivational Strategies for Learning Questionnaire (MSLQ). AnnArbord, MI: NCRIPTAL, The University of Michigan.

ROCES MONTERO, C. y cols. (1999): Relaciones entre motivación, estrategias de aprendizaje y rendimiento académico en estudiantes universitarios. En Mente y Conducta en Situación Educativa. Revista Electrónica del Departamento de Psicología de la Universidad de Valladolid. Vol. I, 1, págs. 41-50.

TEJEDOR, F. J. (COORD.) (1998): Los alumnos de la Universidad de Salamanca: Características y rendimiento académico. Salamanca: Universidad de Salamanca.

UNESCO (1998): Declaración Mundial sobre la Educación Superior en el Siglo XXI: Visión y acción. París: UNESCO.

ZABALZA, M.A. (2000): “Enseñando para el cambio. Estrategias didácticas innovadoras". Ponencia presentada al XII Congreso Nacional y I Iberoamericano de Pedagogía: Hacia el Tercer Milenio. Cambio Educativo y Educación para el Cambio, Tomo I-Ponencias, 26-30 septiembre, Madrid: Sociedad Española de Pedagogía. 